Med Klin Intensivmed Notfmed 2020 · 115:654-667 https://doi.org/10.1007/s00063-020-00740-w Eingegangen: 26. Juli 2020

Angenommen: 25. August 2020

Online publiziert: 12 . Oktober 2020

(c) Springer Medizin Verlag $\mathrm{GmbH}$, ein Teil von Springer Nature 2020

Daniel Kiefl' $\cdot$ Stephan Eisenmann ${ }^{2} \cdot$ Guido Michels $^{3} \cdot$ Mathias Schmid ${ }^{4}$. Corinna Ludwig ${ }^{5} \cdot$ Martin Pin $^{6}$ - Erika Glöckner ${ }^{7}$ Peter-Friedrich Petersen ${ }^{8}$. Domagoj Damjanovic ${ }^{9} \cdot$ Sebastian Schellhaas ${ }^{10}$. Uwe Janssens ${ }^{11}$. Martin Fandler ${ }^{12}$. Sabine Blaschke ${ }^{13}$. Markus Geuting ${ }^{14}$. Thomas Müller ${ }^{15}$. Joseph Menzel ${ }^{16} \cdot$ Alexander Heinzmann ${ }^{17} \cdot$ Matthias Helm ${ }^{18}$. Alexander DinseLambracht $^{19}$ - Michael Bernhard ${ }^{20}$. Sebastian Spethmann ${ }^{21}$. Konrad F. Stock ${ }^{22}$. Dirk-André Clevert ${ }^{23} \cdot$ Raoul Breitkreutz ${ }^{24}$

\title{
Empfehlungen zur Lungen- und Thoraxsonographie bei Patienten mit COVID-19-Erkrankung
}

\section{2-Infektion und der COVID-19- Erkrankung befasst sind, haben über einen transparenten Prozess Empfehlungen zur Lungen- und Thoraxsonographie erarbeitet.}

\section{Einleitung}

Die Lungen- und Thoraxsonographie ist neben der Bildgebung mit der Computertomographie und der Labordiagnostik eine weitere Untersuchungsmodalität bei COVID-19-Erkrankung bei Erwachsenen und Kindern [35, 48]. Die Lungen- und Thoraxsonographie eignet sich zur Erweiterung der klinisch-körperlichen Untersuchung, weil sie sensitiv und gewinnbringend die Lungenoberfläche untersuchen kann. Diese verändert sich mit bislang häufig beschriebenen Mustern in den Sonogrammen bei COVID-19-Pneumonie und im Krankheitsverlauf $[39,48,50,64,66]$. Die Lungen- und Thoraxsonographie ist für diesen Zweck sensitiver als eine Auskultation mit einem Stethoskop [10, 25, 56, 59] und ergänzt die Röntgenverfahren. Darüber hinaus kann bei diagnostizierter $\mathrm{CO}-$ VID-19-Erkrankung die Sonographie bei Verlaufsuntersuchungen genutzt werden $[23,24]$. Aufgrund der SARS-CoV-2Pandemie sind für die Patientenversorgung diagnostische und therapeutische Verfahren zu entwickeln. Berichte aus China und Italien haben auf die mögliche Bedeutung der Thorax- und Lungensonographie aufgrund der prädominan- ten Lungenmanifestationen, insbesondere bei knappen Ressourcen, hingewiesen (Übersicht in [48]).

\section{Methodik}

Die Deutsche Gesellschaft für Interdisziplinäre Notfall- und Akutmedizin e.V. hatte eine Expertengruppe beauftragt, ein Quelldokument $\mathrm{zu}$ spezifischen Informationen der Lungen- und Thoraxsonographie in einem transparenten Prozess vom 24.03.2020 bis zum 16.04.2020 zu erstellen. Auslöser waren erste Berichte aus China und Brasilien [19, 37, 38] aus der klinischen Akut- und Notfallmedizin. Dabei sollten Originalquellen mit Fällen, Fallserien, Konzepten für Einteilungen und Untersuchungsmethoden von internationalen Fachwissenschaftlern sowie multimediale Informationen eingearbeitet werden. Die Empfehlungen wurden daraufhin vom 16.04.2020 bis zum 13.05.2020 mit Delegierten der genannten Fachgesellschaften moderiert und konsentiert. Am 02.06.2020 wurde das Manuskript für eine weitere, unabhängige Begutachtung (Peer-Review) bei einer Fachzeitschrift eingereicht und zuletzt am 25.07.2020 aktualisiert. Experten hatten insgesamt 4 Möglichkeiten, um Vorschläge und Änderungswünsche für den Manuskripttext, inkl. Ergebnisse des Peer-Reviews der Zeitschrift, bis zum Zeitpunkt der Druckfahne anzugeben. Das Dokument beinhaltet zudem einen Überblick über die Grundlagen 


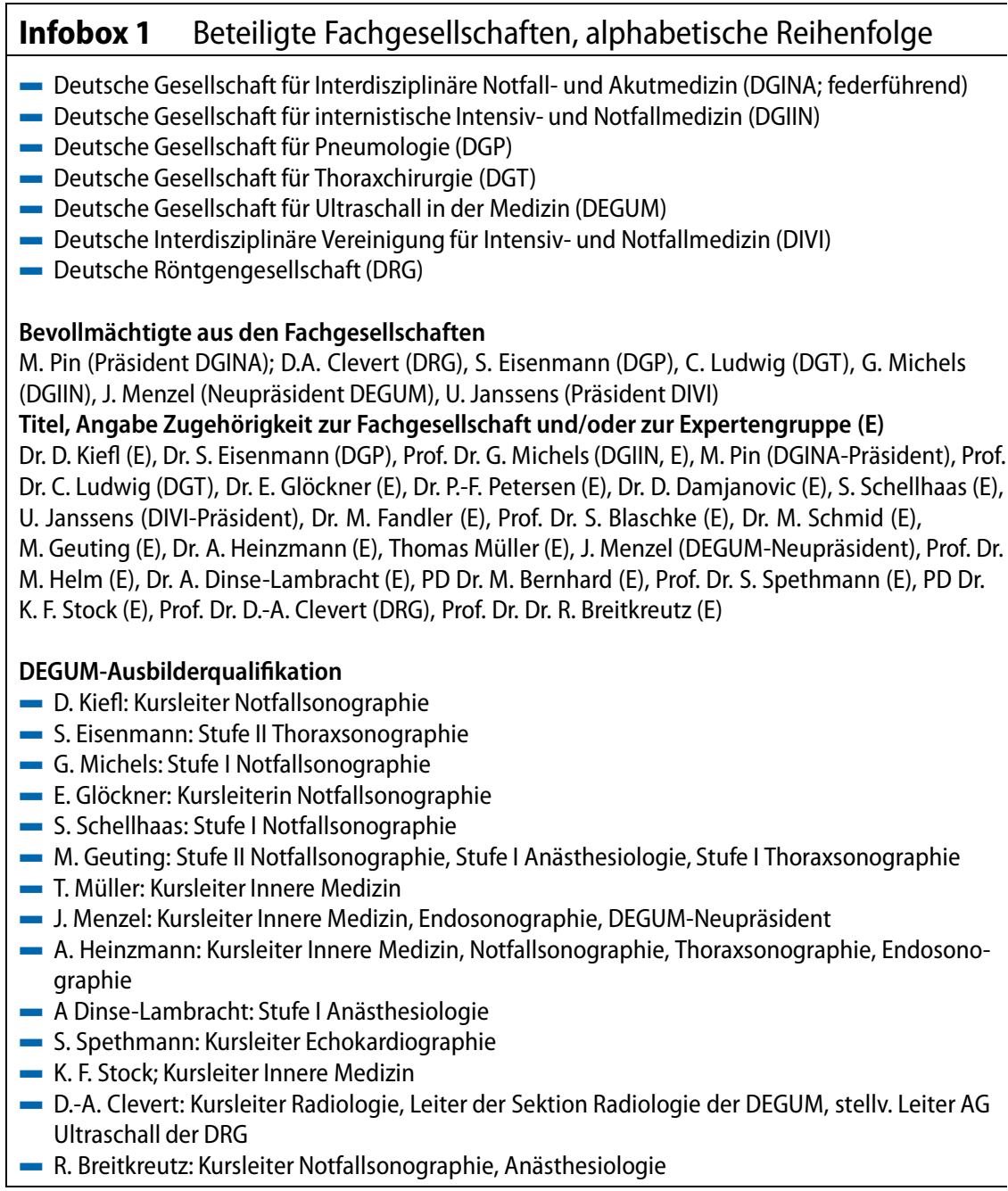

der thoraxsonographischen Diagnostik bei SARS-CoV-2-Pneumonie.

\section{Hintergrund und Ziele}

Die Lungen- und Thoraxsonographie ist eine Untersuchungsmodalität in der Versorgung von Patienten mit COVID-19Pneumonie. Dieses Dokument soll die dazugehörige Wissensvermittlung für die klinische Akut- und Notfall- und Intensivmedizin sowie ambulante Versorgung und mit multimedialen Inhalten unterstützen.

\section{Empfehlungen}

\section{Eigenschutz, Hygiene und Desinfektion („transmission control")}

Der Untersucher soll eine ausreichende persönliche Schutzausrüstung tragen (Kittel, mindestens FFP2-Maske, Handschuhe, Brille, OP-Haube; [3, 20]). Zudem gelten für die Lungen- und Thoraxsonographie die Hygieneleitlinien des RKI [21] und die Empfehlungen der DEGUM [34]. Auch für die Diagnostik sollte ein geeigneter Schallkopfüberzug verwendet werden, der für Interventionen obligat ist [29, 34]. Schallkopf und Kabel sollten vor und nach Untersuchungen wischdesinfiziert werden. Eine ausreichende Einwirkzeit vor Untersuchung des nächsten Patienten sollte eingehalten werden $[23,29,34]$. Das Tragen von

\section{Infobox 2 Anmerkungen}

- Dieses Manuskript wurde bei der AWMF (Arbeitsgemeinschaft der Wissenschaftlichen Medizinischen Fachgesellschaften e.V.) für die Entwicklung des S1-Leitlinien-Sstatus angemeldet (Datum der Anmeldung: 05.08.2020).

- Die Produktneutralität kann nicht vollständig eingehalten werden. Angaben zum Gerät und zu Sonden sind Vorschrift der Befunderhebung. Es gibt viele weitere Hersteller, die mobile oder ultramobile Ultraschallgeräte anbieten.

- Die DEGUM bereitet eine Multizenterstudie zur Thorax- und Lungensonographie vor. Die Studienkoordination liegt bei der TU München (Beratung nach §15 Berufsordnung für Ärzte in Bayern vom 30.04.2020, Ethikkommission der TU München). Informationen folgen auf der Homepage der DEGUM (www.degum.de).

- Die Deutsche Gesellschaft für Allgemeinmedizin (DEGAM) und die Deutsche Gesellschaft für Anästhesie und Intensivmedizin (DGAI) folgten der Einladung zur Gestaltung der Empfehlung nicht. Keine Rückmeldung bis zum Redaktionsschluss kam von der Deutschen Gesellschaft für Innere Medizin (DGIM).

doppelten Handschuhen („double gloving") wurden beschrieben [29]. Tastaturen müssen regelmäßig mit geeigneten Desinfektionsmitteln [13] wischdesinfiziert werden. Verfahrensanweisungen zur Hygiene sollen alle Mitarbeiter kennen und anwenden.

Mobile, akkubetriebene Ultraschallgeräte $[5,54]$ sind aufgrund der besseren Umsetzung von Hygiene/Desinfektion vorzuziehen. Ausgewählte Geräte sollten möglichst getrennt von der Routine ausschließlich für COVID-19-Patienten verwendet werden.

\section{Schallkopfwahl}

Linear-, Konvex- oder Mikrokonvexschallköpfe sind geeignet. Aufgrund der Artefaktwiedergabe sind Konvexschallköpfe mit ausgeschaltetem „Post-processing “-Modus geeignet. Zur genaueren Beurteilung einer fragmentierten Pleura (= Pleuralinie im Sonogramm) können Linearschallköpfe besser geeignet sein. Ein Sektorschallkopf kann mit Einschränkungen für die Nahfeldauflösung Verwendung finden, eignet sich aber besser für die kardiale Untersuchung. 


\section{Untersuchungsweise}

Es bietet sich eine systematische Untersuchungsweise an. Bei bekannten Methoden [15, 43, 49, 52, 62, 65] wird in Regionen (• Abb. 1 und 2; [38, 53]), z. B. von medial nach lateral oder in ähnlicher Weise, untersucht. Eine standardisierte Untersuchungsabfolge wurde für den deutschen Sprachraum von den Ultraschallgesellschaften Deutschlands, Österreichs und der Schweiz vorgeschlagen [53]. Eine korrelierte Lungen- und Thoraxsonographie in Regionen von positiven Befunden einer Thoraxcomputertomographie (Thorax-CT) des [2] wurde vorgestellt [1, 43, 46, 64]. Die Schallkopfführung quer zum Interkostalraum (ICR), in z. B. kraniokaudaler Ausrichtung, dient der sicheren Identifikation der Pleuralinie [15, 52, 65]. Mit längs ausgerichteter Bewegung entlang des Rippenverlaufs („oblique“) kann die einsehbare Lungenoberfläche vergrößert werden [15]. Am häufigsten finden sich positive Befunde in der Lungen- und Thoraxsonographie posterobasal und dorsal [19, 28, 37, 40, 48, 49].

\section{Dokumentation}

Dort, wo positive Befunde erhoben wurden, kann aus praktischen Gründen zur Verlaufsbeurteilung z.B. ein chirurgischer Hautmarkierungsstift mit medizinischer Tinte benutzt werden [43]. Eine standardisierte Befunderhebung wurde durch die deutschsprachigen Ultraschallfachgesellschaften DEGUM/ ÖGUM (Österreichische Gesellschaft für Ultraschall in der Medizin)/SGUM (Schweizerische Gesellschaft für Ultraschall in der Medizin) vorgestellt [53]. Eine papiergebundene Dokumentation erscheint aufgrund des Hygiene-/ Transmissionsrisikos kritisch zu bewerten, insbesondere, wenn Plastikklemmbretter verwendet werden.

\section{Wertigkeit der Lungen- und Thoraxsonographie und Limitationen}

Die Point-of-Care-Lungen- und -Thoraxsonographie [65] eignet sich ggf. zum Verlaufsmonitoring und ggf. Therapie-

Med Klin Intensivmed Notfmed 2020 • 115:654-667 https://doi.org/10.1007/s00063-020-00740-w ๑) Springer Medizin Verlag GmbH, ein Teil von Springer Nature 2020

D. Kiefl · S. Eisenmann · G. Michels · M. Schmid · C. Ludwig · M. Pin · E. Glöckner · P.-F. Petersen · D. Damjanovic - S. Schellhaas - U. Janssens - M. Fandler - S. Blaschke - M. Geuting - T. Müller • J. Menzel · A. Heinzmann · M. Helm · A. Dinse-Lambracht · M. Bernhard - S. Spethmann · K. F. Stock · D.-A. Clevert $\cdot$ R. Breitkreutz

\section{Empfehlungen zur Lungen- und Thoraxsonographie bei Patienten mit COVID-19-Erkrankung}

\section{Zusammenfassung}

Die Lungen- und Thoraxsonographie ist neben der Computertomographie und der Labordiagnostik eine weitere Untersuchungsmodalität bei der COVID-19-Erkrankung. Sie eignet sich zur Erweiterung der klinischkörperlichen Untersuchung, weil sie sensitiv die Lungenoberfläche untersuchen kann. Diese verändert sich in den Sonogrammen mit bislang häufig beschriebenen Mustern bei Patienten mit COVID-19-Pneumonie und im Krankheitsverlauf. Deutsche Fachgesellschaften der klinischen Akut-, Notfall- und Intensivmedizin sowie der Bildgebung, die mit der Versorgung von Patienten mit SARS-CoV-2-Infektion und der COVID-19-Erkrankung befasst sind, haben unter der Führung der Deutschen Gesellschaft Interdisziplinäre Notfall- und Akutmedizin e. V. (DGINA) Empfehlungen zur Lungenund Thoraxsonographie abgestimmt. Das
Dokument wurde über einen transparenten Prozess durch eine Expertengruppe und Delegierte der Fachgesellschaften erstellt. Quellen der ersten internationalen 200 Fallanalysen wurden zusammengefasst. Bislang häufig beschriebene thoraxsonographische Muster werden vorgestellt. Anhand von Fallbeispielen und multimedialen Inhalten soll das Dokument die Anwender unterstützen und Qualitätsmerkmale und das Potenzial der Thorax- und Lungensonographie aufzeigen. Die Deutsche Gesellschaft für Ultraschall in der Medizin e. V. (DEGUM) führt dazu eine Multizenterstudie durch (Studienkoordination TU München).

Schlüsselwörter

Pneumonie · Point-of-Care Ultraschall (POCUS) · Lunge · Ultraschall · SARS-CoV-2

\section{German recommendations on lung and thoracic ultrasonography in patients with COVID-19}

Abstract

Lung and chest ultrasound are further examination modalities in addition to computed tomography and laboratory diagnostics in patients with COVID-19. It extends the clinical-physical examination because it can examine lung surface sensitively. Lung surface pattern changes have been found in sonograms of patients with COVID-19 pneumonia and during the course of the disease. German specialist societies of clinical acute, emergency and intensive care medicine as well as imaging, which are concerned with the care of patients with SARS-CoV-2 infection and COVID-19, have coordinated recommendations for lung and thorax sonography. This document has been created within a transparent process, led by the German Society of Interdisciplinary Emergency and Acute Medicine e.V.
(DGINA), and worked out by an expert panel and delegates from the societies. Sources of the first 200 cases were summarized. Typical thorax sonographic findings are presented. International sources or standards that were available in PubMed until May 24, 2020 were included. Using case studies and multimedia content, the document is intended to not only support users but also demonstrate quality features and the potential of chest and lung sonography. The German Society for Ultrasound in Medicine (DEGUM) is carrying out a multicenter study (study coordination at the TU Munich).

Keywords

Pneumonia $\cdot$ Point-of-Care Ultrasound (POCUS) · Lung · Ultrasound · SARS-CoV-2 

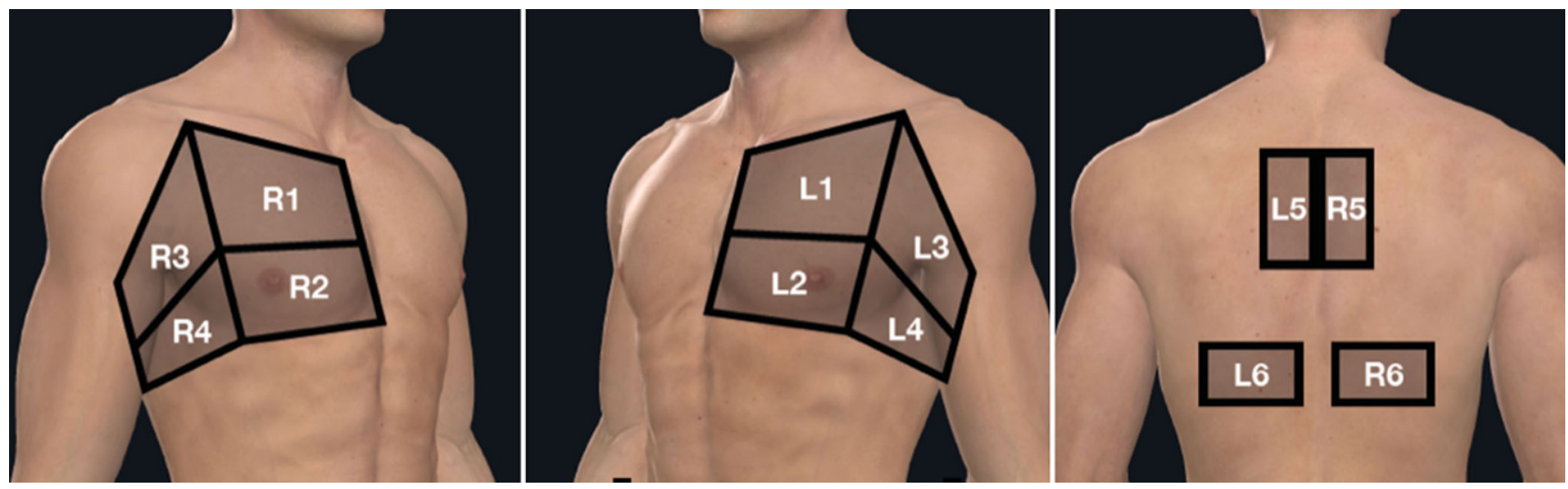

Abb. 1 ॥ Standardisierbare Untersuchungsregionen für die Beurteilung der Lungenoberfläche. (Nach Monteiro Pereira et al. WINFOCUS, Brasilien; mit freundl. Genehmigung aus [38])

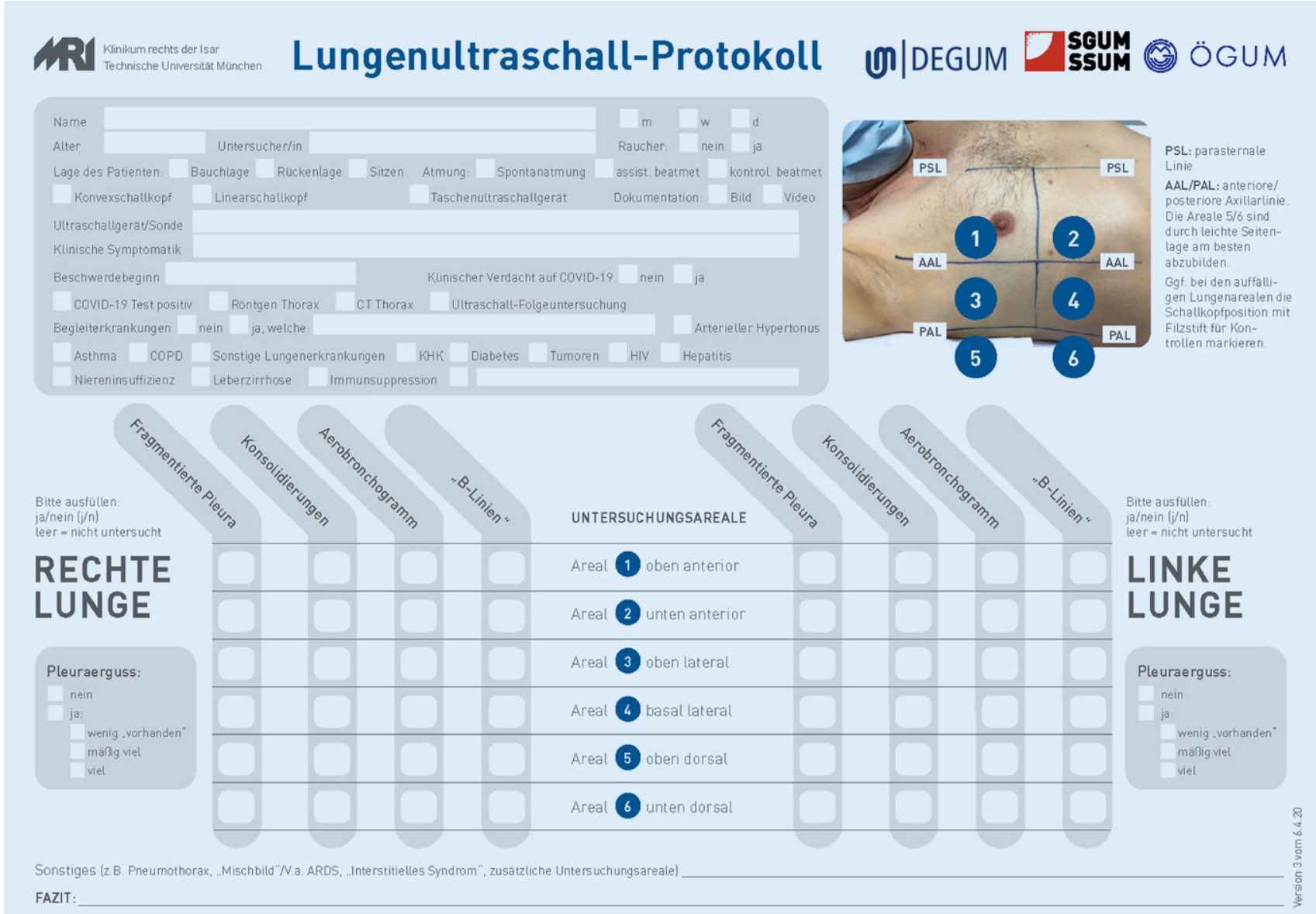

Abb. 2 \ Standardbefunddokumentation nach K.F. Stock, TU München, die von der DEGUM/ÖGUM (Österreichische Gesellschaft für Ultraschall in der Medizin)/SGUM (Schweizerische Gesellschaft für Ultraschall in der Medizin) gestützt wird [53]. Das Dokument darf nichtkommerziell gedruckt werden und ist auf den Homepages der Fachgesellschaften verfügbar. (৫) K.F. Stock, TU München, alle Rechte vorbehalten) 


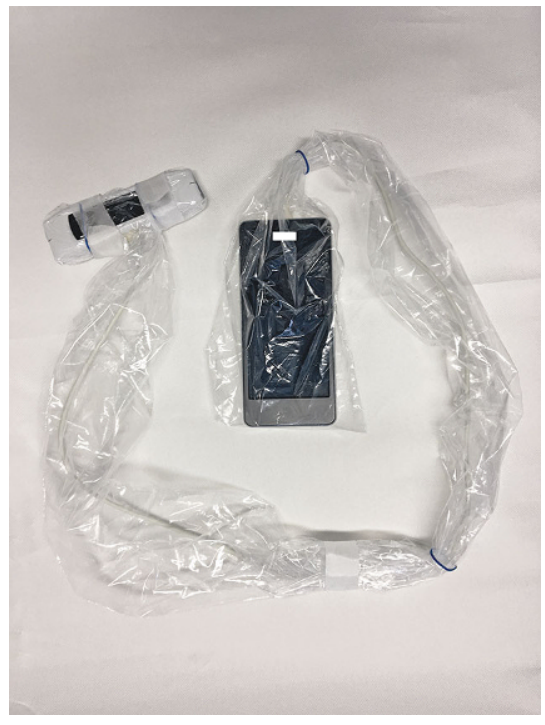

Abb. 3 A Eingepacktes ultraportables Ultraschallgerät

steuerung bei COVID-19-Patienten [23, 24, 29, 48, 64] sowie zur Diagnostik alternativer bzw. ergänzender Diagnosen $[9,42]$. Hauptgrund sind periphere pleuraassoziierte Befunde, die mit CTBefunden wie „Milchglastrübungen“ und Konsolidierungen korrelieren [1, 2, 19, 28, 29, 37, 40, 43, 46, 49]. Eine Fallsammlung aus München wurde kürzlich vorgestellt $[8,44,45,54]$. Die Lungen- und Thoraxsonographie kann keine zentralpulmonalen Befunde erheben, weil sie nicht an die Pleura heranreichen, aber die diagnostische Sicherheit erhöhen $[43,65]$. Die Lungen- und Thoraxsonographie sollte aber nicht isoliert zum allgemeinen Screening oder zur Diagnosestellung von COVID19 benutzt werden. Die Lungen- und Thoraxsonographie wurde für die Triage beim Massenanfall von Schwerkranken kommentiert [12].

Eine Schweregradbestimmung des Zustands bei COVID-19-Pneumonie ist über die Lungen- und Thoraxsonographie begrenzt möglich. Es kann unterschieden werden zwischen interstitiellen Veränderungen (vermehrte B-Linien), Konsolidierungen und Konsolidierungen mit Aerobronchogrammen [8, 19, 28, 29, 37, 40, 43, 44, 48, 49, 62]. Es zählt die klinische Bewertung. Eine Therapiesteuerung ist nur im Rahmen der allgemeinen Intensivtherapie $\mathrm{zu}$ empfehlen (Flüssigkeitsmanagement, Recruitment- manöver, Weaning mit Zwerchfellsonographie etc.). Wiederholte patientennahe Untersuchungen sollten aus hygienischer Sicht eher minimiert werden, sofern sie nicht im Rahmen unbedingt erforderlicher oder wissenschaftlicher Tätigkeiten durchgeführt werden [3].

\section{Ergänzende Untersuchungen}

Alternative oder weitere Diagnosen (Herzinsuffizienz, Pneumothorax, bakt. Pneumonie, Thrombose und Lungenarterienembolie, Hypovolämie etc.) sind zu prüfen. Dies ist auch schon bei der ersten Sonographie möglich. Die fokussierte Beurteilung des Volumenstatus und der linksventrikulären Herzfunktion sollte mit einem Schallkopfschwenk auf die Vena cava inferior (VCI) und das Herz von subkostal durchgeführt werden $[36,43]$. Die Anlotung der VCI in der kurzen Achse ist wegen der sicheren Beurteilung des Durchmessers v. a. bei Hypovolämie geeignet.

\section{Häufig beschriebene Sonogram- me für die Mustererkennung}

Einfache B-Bild-Sonogramme ohne Zusatzfunktionen, auch von älteren Geräten, sind ausreichend $[43,65]$. Beschrieben wurden Pleuraunregelmäßigkeiten (Verdickungen, Unterbrechungen), fokal-regionale multiple B-Linien, einzeln stehende B-Linien, abwechselnde multiple, verschmelzende B-Linien, das sog. „Wasserfallzeichen“, ausgesparte „normale“ Areale sowie kleinere und größere subpleurale Konsolidierungen. Kleinere Ergüsse sind möglich [8, 19, 28, 29, 37, $40,43,44,49,62]$.

\section{Lern- und Trainingsaufwand}

Pleuraergüsse, B-Linien-Diagnostik und Untersuchungsweise als Bestandteile der Lungen- und Thoraxsonographie gelten für Anfänger mit Grundkenntnissen als auch für Ultraschallerfahrene als leicht erlernbar [4, 7, 43, 48, 52]. Lernkurven für Differenzialbefunde der Pleura/ Pleuralinie und Konsolidierungen wurden bisher nicht überprüft. Daher sollten diese Befunde supervidiert werden.

\section{Anmerkungen und Hintergründe}

\section{Indikationen für die Lungen- und \\ Thoraxsonographie}

\section{Generell für Praxen, Notaufnahme/ Intensivstation}

- Husten, Dyspnoe, Sauerstoffbedarf, Schock

- Fieberfreie Patienten mit atypischem Thoraxschmerz

- Differenzialdiagnose, alternative Befunde oder Diagnosen wie z. B. Pleuraerguss, Empyem und Pneumothorax, Infiltrate, Lungenödem u.v.a.

- Intervention, z. B. Ergusspunktion

- Diagnostik von Funktionen (Zwerchfell, Lungengleiten)

- Verlaufskontrollen

\section{Speziell für die Intensivstation}

- Differenzialdiagnose, alternative Befunde wie z. B. Pleuraerguss und Pneumothorax.

- Bei schneller klinischer Verschlechterung des Patienten, wenn eine CTFahrt gefährdend wäre, um z. B. wichtige alternative Diagnosen (z. B. Pneumothorax) auszuschließen oder Hinweise zu finden (kein Lungengleiten). Dies hätte eine therapeutische Konsequenz mit Punktion zur Folge. - Z.B. mit dem Augenmerk auf die Zunahme der basalen Konsolidierungen als Hinweis für eine Befundverschlechterung, um ggf. Thorax-CT-Ressourcen einzusparen $[24,33]$.

\section{Wo und wann kann die Lungen- und Thoraxsonographie in der Versorgung eingesetzt werden?}

Die Lungen- und Thoraxsonographie ist, insbesondere bei Nutzung von mobilen, akkubetriebenen Ultraschallgeräten, sofort verfügbar (•Abb. 3). Sie kann als Erweiterung der klinischen Untersuchung zur Beurteilung der Lungenoberfläche jederzeit genutzt werden, da die Auskultation mit dem Stethoskop weniger aussagekräftig ist.

Daher kann die Lungen- und Thoraxsonographie als Ergänzung zur Tho- 
rax-CT klinisch an verschiedenen Stellen der Versorgung (Praxen, ÄBD, Ambulanzen, Notaufnahme, IMC, Intensivstation) eingesetzt werden [6]. Es besteht keine Strahlenbelastung [14].

An folgenden Stellen in der Diagnostik wäre der Einsatz der Sonographie denkbar:

\section{- in der Notaufnahme:}

- als Erstdiagnostik: bei Kindern, Schwangeren, gebärfähigen Frauen, wenn die Thorax-CT-Indikation enger gestellt werden muss;

- wenn kein CT vorhanden ist;

- falls eine Patient aus klinischer Sicht nicht aufgenommen werden soll zur Erweiterung der körperlichen Untersuchung;

- zur Indikationsstellung der Thorax-CT, falls die Abwägung von Ressourcen erforderlich ist;

- auf der COVID-19-Krankenstation

(Isolier-oder Überwachung):

- bei zunehmendem Sauerstoffbedarf;

- Intensivstation:

- frühestens nach der ersten ThoraxCT im Verlauf;

- zur Indikationsprüfung für weitere CT-Verlaufsuntersuchungen bei Beatmung;

- bei „klinischer Verschlechterung“, Beatmung, Umlagerung - falls der klinische Zustand zu kritisch für einen Transport zur Thorax-CT ist;

- für Routineverlaufsuntersuchungen, Wiederbelüftung oder Zunahme der Konsolidierungen (stärkere Konsolidierungen $=$ Verschlechterung oder Wiederbelüftung $=$ Verbesserung).

\section{COVID-Praxen, Hausarztpraxen, ärztliche Bereitschaftsdienste der ambulanten Versorgung}

Aktuell wird die Untersuchungsmethode für die breite hausärztliche Versorgung als zu speziell angesehen. Daher beziehen wir uns auf die Schnittstellen zur Versorgung am Krankenhaus (Notaufnahme, Intensivstation). Für die Praxen mit Ultraschallgeräteausstattung können allerdings die Empfehlungen sinngemäß angewendet werden. Damit könnte eine ambulant-häusliche Untersuchung oder ambulanten Verlaufsbeurteilun- gen und Vernetzung in der ambulanten Versorgung zwischen Notaufnahmen, ärztlichen Bereitschaftsdiensten und hausärztlicher Versorgung ermöglicht werden $[47,60]$. Befunderhebungen im ambulanten Verlauf, vom Auftreten bislang häufig beschriebenen Mustern bis zur Normalisierung der Lungenoberfläche, wurden z.B. für 3 SARS-CoV-2infizierte Ärzte in Quarantäne als häusliche Selbst- und Verlaufsbeurteilung demonstriert [47].

\section{Sind positive Befunde an den Schweregrad gebunden?}

Man sollte zwischen diagnostischem und klinischem Schweregrad unterscheiden. Ein PCR-Test beinhaltet ein binäres Ergebnis. Für die CT-Diagnostik liegen radiologische Einteilungen vor [2]. Beide Methoden beinhalten noch keine Information zur Bestimmung eines klinischen Schweregrads.

Für die Thorax- und Lungensonographie liegen Konzepte für eine Schweregradeinteilung der Befunde vor [66] oder sonographische Graduierung [48] nach Grad der Konsolidierung (Pleuraverdickung, B-Linien, Mikrokonsolidierungen, kleine und größere subpleurale Konsolidierungen sowie kleinere Ergüsse).

Ein klinischer Schweregrad wurde Befunden der Thorax- und Lungensonographie im Bereich der Intensivmedizin bisher von Tan et al. bei $n=12$ Fällen bzw. bei Verlaufsuntersuchungen mit COVID-19-Erkrankung und in $n=10$ von Dargent et al. zugeordnet [11, 55]. Der „lung ultrasound score“ (LUSS) ließ sich gut mit dem Krankheitsverlauf und dem Einsatz von Beatmungsformen korrelieren [11]. Der „modifizierte Lungenultraschallscore“ (MLUS) wurde mit dem jeweiligen CT-Verlauf verglichen und es konnten 3 Schweregradgruppen abgeleitet und unterschieden werden [55]. Zudem wurde gezeigt, dass die Befunde der Thorax- und Lungensonographie im Vergleich zu $n=20$ SARSCoV-2-negativen Patienten mit „community acquired pneumonia“ (CAP) sich (ausreichend gut) hinsichtlich des Musters im Sonogramm unterscheiden ließen [55].

\section{Verlaufsuntersuchungen}

Die bislang häufig beschriebenen positiven Befunde in der Thorax- und Lungensonographie wurden hauptsächlich posterobasal lokalisiert [48]. Sie sind damit gut für Verlaufsuntersuchungen im Liegen zugänglich. Wenn die Regionen der Schädigungen der Lungenoberfläche bereits durch eine Thorax-CT-Untersuchung beschrieben wurden [2], besteht die häufig vorgestellte Möglichkeit einer CT-korrelierten Verlaufsuntersuchung $[14,19,23,28,29,43,68]$.

Weiterhin vermutet man für die beobachteten subpleuralen Konsolidierungen periphere Lungeninfarkte aufgrund von Venenthrombosen $[26,51,57,67,69]$ bisher ungeklärter Ätiologie. Exemplarisch wurde dies mittels technisch aufwändigerem thorakalem Kontrastmittelultraschall („contrast-enhanced ultrasound“, CEUS) bestätigt [58].

Die Lungen- und Thoraxsonographie hat im Rahmen der Lungenarterienemboliediagnostik bereits eine etablierte und wissenschaftlich begründete Indikation und ihren entsprechenden Stellenwert [30, 31, 41]. Es gibt für das Thema venöse Thrombembolien eine deutschsprachige Leitlinie für die „Venenthrombose und Lungenembolie" mit Hinweisen auf die thoraxsonographische Diagnostik der peripheren Lungenembolie [18].

\section{Schutz des Personals, persönliche Schutzausrüstung, Hygiene, Desinfektion, Überzüge}

Es gibt aktuelle Empfehlungen zur allgemeinen Hygiene $[3,21,34]$ und Kontagiosität [61] und Hinweise für die Lungen- und Thoraxsonographie [29, $32,48]$ für die Anwendung bei COVID19-Patienten. In den Gesundheitseinrichtungen der Autoren gibt es offenbar heterogene Anweisungen, wie damit umzugehen ist. In einer Laborstudie wurde gezeigt, dass SARS-CoV-2 an Oberflächen und in Aerosolen mehrere Tage kontagiös bleibt [27, 61]. Daher könnte man vermuten, dass das Virus an der Körperoberfläche oder den Haaren kontagiös bleiben kann. Das SARS-CoV-2 wurde als hochkontagiös 
betrachtet und die vollständige persönliche Schutzausrüstungen (PSA) während der Anwendung der Sonographie beschrieben [29]. Bei unvermeidbarem Nahfeldkontakt im Rahmen der Ultraschalluntersuchung sollte auf eine intakte und konsequente persönliche Schutzausrüstung geachtet werden $[3,20,29,48]$. Hinweise der US-amerikanischen Gesellschaft für Echokardiographie wurden kürzlich formuliert [3]. Dies führte zur nichteinheitlichen Bewertung der PSA für eine Lungen- und Thoraxsonographie, z.B. im Hinblick auf doppelte Handschuhe („double gloving"), OPHaube oder Schallkopfüberzug [3, 5, 28, 29]. Die Leitlinien des RKI [21] aus dem Jahr 2011 und die der DEGUM aus 2018 [34] beinhalten für die Sonographie jedoch beide keine Angaben zu einer Virus- oder SARS-CoV-2Pandemie. Zur Desinfektion sind Mittel mit nachgewiesener Wirksamkeit, mit dem Wirkungsbereich „begrenzt viruzid“, wirksam gegen behüllte Viren anzuwenden [13]. Die Frage, wie lange SARS-CoV-2 am menschlichen Körper haften kann und infektiös ist, bleibt aktuell weiterhin ungeklärt. Daher steht bei der Mehrheit der Autoren eine konservative Vorgehensweise, d.h. die vollständige persönliche Schutzausrüstung [29], im Vordergrund. Die Lungenund Thoraxsonographie sollte gemäß den lokalen Hygienestandards durchgeführt werden. In einigen Einrichtungen kann das Sicherheitsniveau der erforderlichen PSA vom Risikoniveau des Patienten in Bezug auf SARS-CoV-2 abhängen (nichtvermutet $=$ minimales Risiko, vermutet $=$ mittleres Risiko, bestätigt $=$ hohes Risiko). In einigen Institutionen werden Verdachtsfälle und bestätigte Fälle ähnlich behandelt. Bei der Untersuchung ist der Eigenschutz besonders zu beachten. Der nichtintubierte Patient sollte eine Maske tragen. Vorsicht ist bei hustenden Patienten geboten, daher sollte die Indikation und Durchführbarkeit der Ultraschalluntersuchung kritisch überprüft werden.

Es sollte erwogen werden, Personal, das besonders anfällig für schwere Komplikationen von COVID-19 sein könnte (beispielsweise Untersucher über $>60$ Jahre, chronische Krankheiten, Im- munschwäche oder Schwangere), je nach den örtlichen Anweisungen nicht für die Lungen- und Thoraxsonographie bei Patienten einzusetzen.

\section{Klinische Untersuchung vs. Lungen- und Thoraxsonographie}

Die klinische Untersuchung bei COVID19-Erkrankung ist unspezifisch [17]. Die Lungen- und Thoraxsonographie istesim Grundsatz bisher auch $[6,8,19,28,29$, $37,40,43-45,48,49,53,62]$ und kann keine Viruspneumonie diagnostizieren. Sie kann aber minimale Veränderungen deutlich sensitiver erkennen als die körperliche Untersuchung und Auskultation. Pathologische Befunde mit bislang häufig beschriebenen Mustern im Sonogramm bei COVID-19-Patienten wurden beschrieben $[6,8,19,28,29,37,40$, 43-45, 48, 49, 53, 62]. Der Mehrwert einer Lungen- und Thoraxsonographie im Rahmen der körperlichen Untersuchung bei pleuropulmonalen Erkrankungen ist evidenzbasiert [65].

\section{Computertomographie vs. Lungen- und Thoraxsonographie}

Die Computertomographie (CT) ist eine sensitive Bildgebung bei COVID-19 [1,2, 46]. Eine Übersicht von Lomoro et al. [28] hat 26 Publikationen mit Thorax-CTDiagnostik und jeweils mehr als 10 Fällen pro Publikation zusammengefasst dargestellt. Retrospektiv wurde auch die Lungen- und Thoraxsonographie den Ergebnissen der Thoraxröntgenuntersuchung und CT-Ergebnissen einer Fallserie gegenübergestellt [28]. Für die Thorax-CT wurden verschiedene Muster, die auch von anderen Viruspneumonien bekannt sind, beschrieben (Milchglas, "crazy paving", beidseitige Verteilung, oft pleuraständig, dorsobasal; [1, 2, 28, 46]). Die peripheren Befunde der CTUntersuchungen können auch in der Lungen- und Thoraxsonographie mit hoher Übereinstimmung gefunden werden $[14,19,23,28,29,43,68]$. Lu et al. hatten Befunde der Lungen- und Thoraxsonographie mit CT-Korrelationen als probate Methode in 30 Fällen dargestellt [29]. Die CT-Korrelationen könnten sich gut für die Verlaufsuntersuchungen eig- nen [23]. Computertomographen und Ultraschallgeräte müssen nach COVID19-Verdacht oder -Diagnose desinfiziert werden [2]. Mittels Lungen- und Thoraxsonographie könnten notwendige Ressourcen für die Bildgebung ggf. rationeller eingesetzt werden $[5,43$, 49]. Die Lungensonographie kann und soll die Thorax-CT-Untersuchung nicht ersetzen. Sie soll als ergänzende Untersuchungsmodalität die Möglichkeiten der körperlichen Untersuchung erweitern. Die Studienlage zur Lungen- und Thoraxsonographie sollte in 6-12 Monaten erneut überprüft werden.

\section{Sonographische Technik und Ergänzende sonographische Verfahren}

Für das optimale B-Bild zu Erkennung von Lungenartefakten sollte ein Preset verwendet werden, dass Bildoptimierungen wie „tissue harmonic imaging" (THI) und "specle reduction imaging" (SRI) ausschaltet. Bei soliden Prozessen wie Konsolidierungen kann die Nutzung des THI-Modus von Vorteil sein. Die beiden Presets sollten im Gerät für COVID-19Untersuchungen hinterlegt sein. Bei den Herstellern kann ggf. für die Speicherung der Presets angefragt werden. Regionen, Zonen sowie Quadranten sind synonym gebräuchliche Beschreibungen der Lokalisationen, in denen der Schallkopf geführt wird. Radiologisch kann in Ober-, Mittel- und Unterfeld eingeteilt werden. Zur bildlichen Veranschaulichung für den Lernenden für den Vorschub des Schallkopfs wurde die „Rasenmähertechnik" als Begrifflichkeit [43] oder „mäanderförmig“ vorgestellt.

Die schrägen Anlotungen der Schallebenen („oblique windows") entlang des Rippenverlaufs vergrößern die Fläche der einsehbaren Pleura, weil Rippenartefakte mit dorsaler Schallauslöschung umgangen werden, und erweitern damit die Nutzbarkeit der Methode. Zudem können Kippbewegungen das Blickfeld erweitern. Ausgenommen sind die Regionen L5 und R5, wo man longitudinal (gemeint ist hier der Bezug zur Körperlängsachse) anloten sollte [15]. Cave: In Deutschland wird der Querschnitt meist als der Bezug zur Rippe, „bat sign“, im 

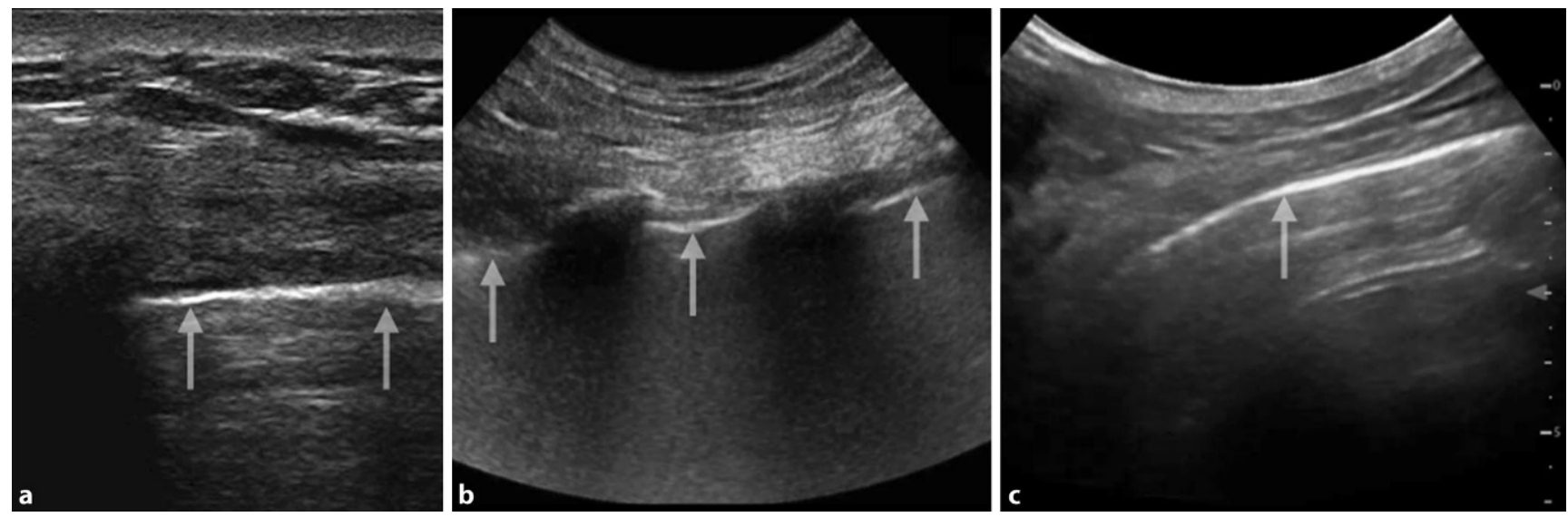

Abb. 4 ム Sonogramm einer normalen Lungenoberfläche mit Reflexmuster beider Pleurablätter (im Folgenden „Pleuralinie“ genannt). a Linear- und b,c Konvexschallkopf. b „Bat sign“. c Pleura longitudinal zum Rippenverlauf ohne dosale Schallauslöschungen

Sinne der kraniokaudalen Ausrichtung erklärt, aber von Gargani und Volpicelli mit „longitudinal“ bezeichnet [15].

International wurden teils divergierende Konzepte zu den Untersuchungsmethoden mit LU/LUS bei COVID-19 vorgestellt [15, 48, 52]. Diese enthalten allerdings keine klinischen Daten. Soldati et al. und Stock et al. schlugen eine Standardisierung der Befunderhebung vor und wiesen auf die jeweilige italienische und deutsche Studiendatenbank hin $[49,53]$. Für Deutschland ist eine Multizenterstudie, führend durch Koautor PD Dr. Stock bereits geplant (Ethikvotum vom 30.04.2020 an der TU München).

\section{Alternative Diagnosen, Pneumothorax}

Ein Pneumothorax ist eine wichtige alternative Diagnose bei klinischen Symptomen [42] auch bei nichtbeatmeten Notfallpatienten. Bei Beatmung von COVID-19-Patienten sollte bei Verschlechterung oder vitaler Bedrohung auch ein Pneumothorax als alternative Diagnose in Betracht gezogen werden. Dieser kann vor einem CT-Transport leitliniengerecht mit der Lungen- und Thoraxsonographie untersucht werden [42].

Eine präklinische Lungen- und Thoraxsonographie kann bei Verfügbarkeit von Ultraschallgeräten auch zur Ermittlung alternativer Diagnosen vorgenommen werden.
Mittels B-Linien-Diagnostik kann ein häufiges kardial bedingtes Lungenödem von einem selteneren ARDS differenziert werden [9]. Beim kardial bedingten Lungenödem sind B-Linien atemverschieblich und in den gravitationsunabhängigen Arealen (meist ventral) zu finden. Bei COVID-19-Pneumonie und ARDS wurden dagegen unbewegte B-Linien, Unterbrechungen der Pleura und von B-Linien ausgesparte Areale (,spared areas") in den abhängigen Arealen (posterobasal) beschrieben, sodass für die COVID19-Pneumonie bislang häufig beschriebene Musterbefunde in Abwechslung mit normalem Befund erhoben werden. Dies ist beim kardial bedingten Lungenödem nicht der Fall.

\section{Notfall-TTE - Myokarditis und Herzfunktion}

Eine TTE bzw. Notfall-TTE kann mit einem Sektorschallkopf mit höherer Qualität durchgeführt werden. Dieser kann auch für die B-Linien-Diagnostik in der LUS benutzt werden, eignet sich aber wegen der geringeren Nahfeldauflösung weniger für die Beurteilung der Lungenoberfläche oder zum Nachweis von Konsolidierungen. Viruserkrankungen können zu einer Myokarditis führen. Hauptziel ist die geringste Exposition [3]. Die Untersuchungsweise kann entlang aktueller Empfehlungen erfolgen und nach klinischem Kontext, sodass die Schallfenster parasternal, subkostal und apikal zur Verfügung stehen [36, 63]. Die VCI kann auch aus einem modifizier- ten 4-Kammer-Blick heraus erzeugt werden. Der zentrale Anteil sollte zwischen Zwerchfell und Höhe der Lebervenen mit Durchmesser, aber auch hinsichtlich der Pulsation und Atemvariabilität beurteilt werden [36, 63].

Für COVID-19 wurde auf einen Fall hingewiesen, der als kardiale Manifestation ohne Lungenbeteiligung gewertet wurde [22].

\section{Thrombose - Lungen- arterienembolie}

Aktuell wurde auf eine mögliche thromboembolische Pathophysiologie hingewiesen [26, 51, 57, 67, 69]. Sonogramme für periphere Lungeninfarkte haben Ähnlichkeiten zu Befunden, die bei COVID-19 beschrieben wurden [26, 51, $57,67,69]$. Der popliteale und femorale 2-Punkte-Kompressionsultraschall des Beins [18] kann zum Ausschluss einer Thrombose und bei positivem Befund als ein Hinweis für eine Lungenarterienembolie/Infarktpneumonie dienen.

\section{Bisherige Evidenz für die Lungen- und Thoraxsonographie durch Fallstudien}

Die größte Serie mit $n=58$ Fällen wurde von Lomoro et al. [28] vorgestellt. Fallserien mit vergleichbaren Ergebnissen wurden beschrieben $[28,29]$ und zusammenfassend analysiert [48].

Eine der ersten Fallpräsentationen eines COVID-19-Patienten aus Rom, Italien, beschrieb eine irreguläre Pleu- 

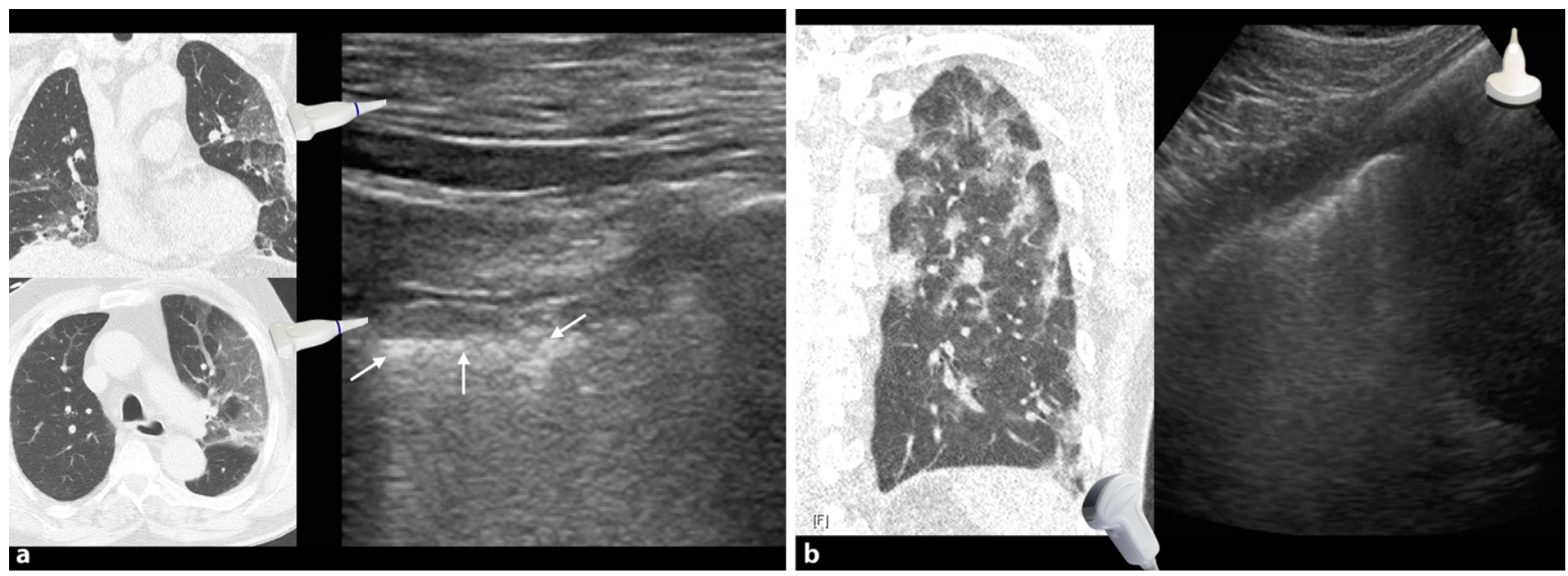

Abb. 5 \ Muster einer verdickten, unregelmäßigen Pleuralinie (Pfeile); oben Linear- und unten Konvexschallkopf. (Mit freundl. Genehmigung Dr. M. Schmid, München)

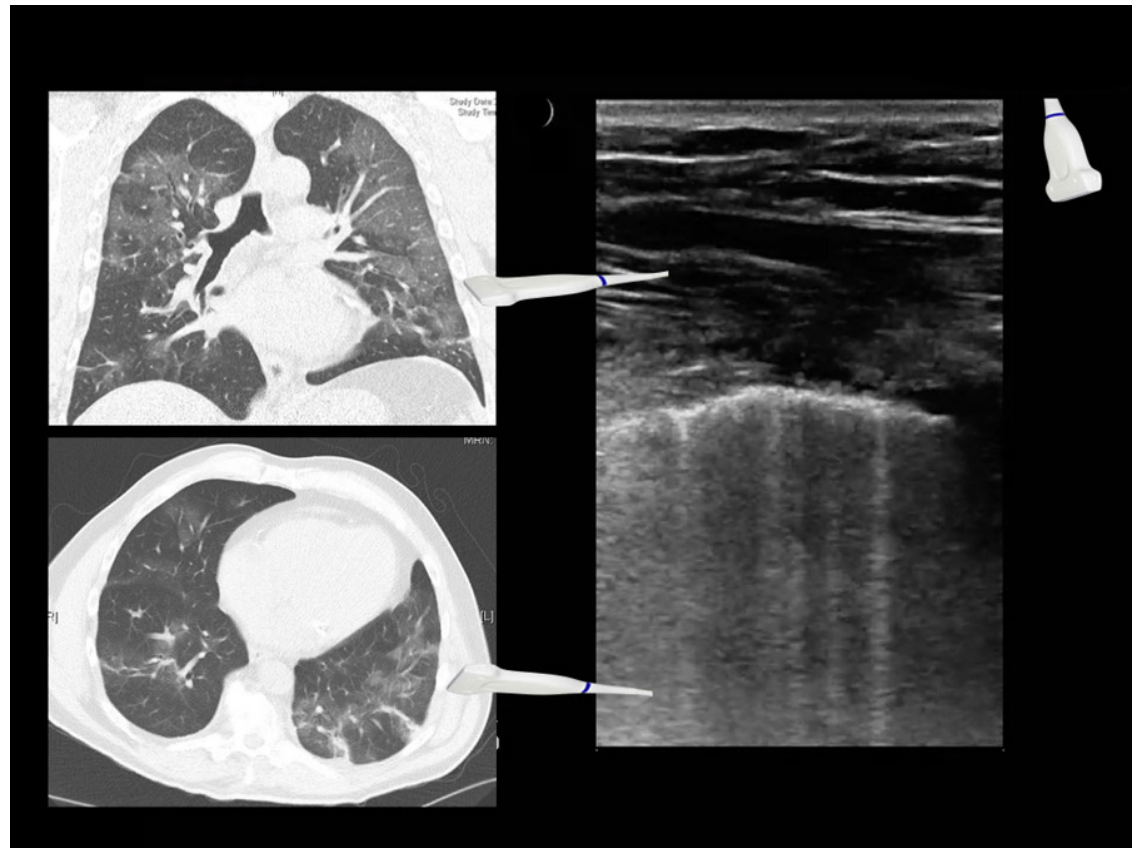

Abb. 6 \ Muster einer unruhigen, fragmentierten Pleuralinie mit Mikrokonsolidierungen, vereinzelte B-Linien; Linearschallkopf, longitudinale Anlotung der Lungenoberfläche. (Mit freundl. Genehmigung Dr. M. Schmid, München)

ralinie, subpleurale Mikrokonsolidierungen und verschmelzende B-Linien [6]. Das Vorgehen nach Rouby wurde „protokollbasiert“ benutzt, um die Lungenfenster superior/inferior, anterior/ lateral/posterior, rechts/links zu untersuchen $[38,52]$.

In einer Fallserie mit 20 Patienten aus Changsha und Peking, China, wurden unregelmäßige Pleuralinien, variable B-Linien-Muster (fokal, multifokal, konfluierend), Konsolidierungen mit vamit Airobronchogrammen) und A-Linien (= Reverberationen der Pleuralinie) in der Phase der klinischen Erholung gefunden. Pleuraergüsse wurden weniger häufig gesehen [37].

In einer Fallserie mit 20 Patienten aus Xi'an, China, benutzten die Autoren die jeweils gleiche Untersuchungsmethode und gaben an, dass $75 \%$ der Patienten positive Befunde in den posterorinferioren Regionen haben. Verschmel- zende B-Linien „Wasserfallzeichen“, irreguläre Pleuralinien, Mikrokonsolidierungen und Konsolidierungen mit Aerobronchogrammen wurden beschrieben [19].

In 12 Fällen aus Piacenza, Italien, wurden diffuse B-Linien-Muster mit abwechselnd normalem Lungensonogramm gefunden [43, 48, 66], die „spared areas“, die für das ARDS beschrieben wurden [9]. Drei Patienten hatten posteriore Mikrokonsolidierungen.

Zwei Fälle aus Lucca, Italien, beschrieben bei intermediärer Pneumonie Mikrounterbrechungen der Pleuralinie und Mikrokonsolidierungen mit großen echogenen, vertikalen Artefakten, die von diesen Unterbrechungen ausgehen [49]. Dargent et al. und Tan et al. stellten die ersten Fallserien bei COVID-19-Erkrankung mit Verlaufsuntersuchungen für den Lungenultraschall mit $n=10$ und $n=12$ Patienten vor $[11,55]$.

Daher wurde der Versuch unternommen, bislang häufig beschriebene Muster der Veränderungen der Lungenoberfläche für den Krankheitszustand bei COVID-19 zu definieren [48].

\section{Bislang häufig beschriebene}

Muster in der Lungen- und Thoraxsonographie bei COVID-19

Aus 5 bestätigten COVID-19-Fällen werden dazu exemplarisch die Muster der Lungen- und Thoraxsonographie von Originalbefunden eines mobilen Point-of-Care-Ultraschallgerätes (CX50 


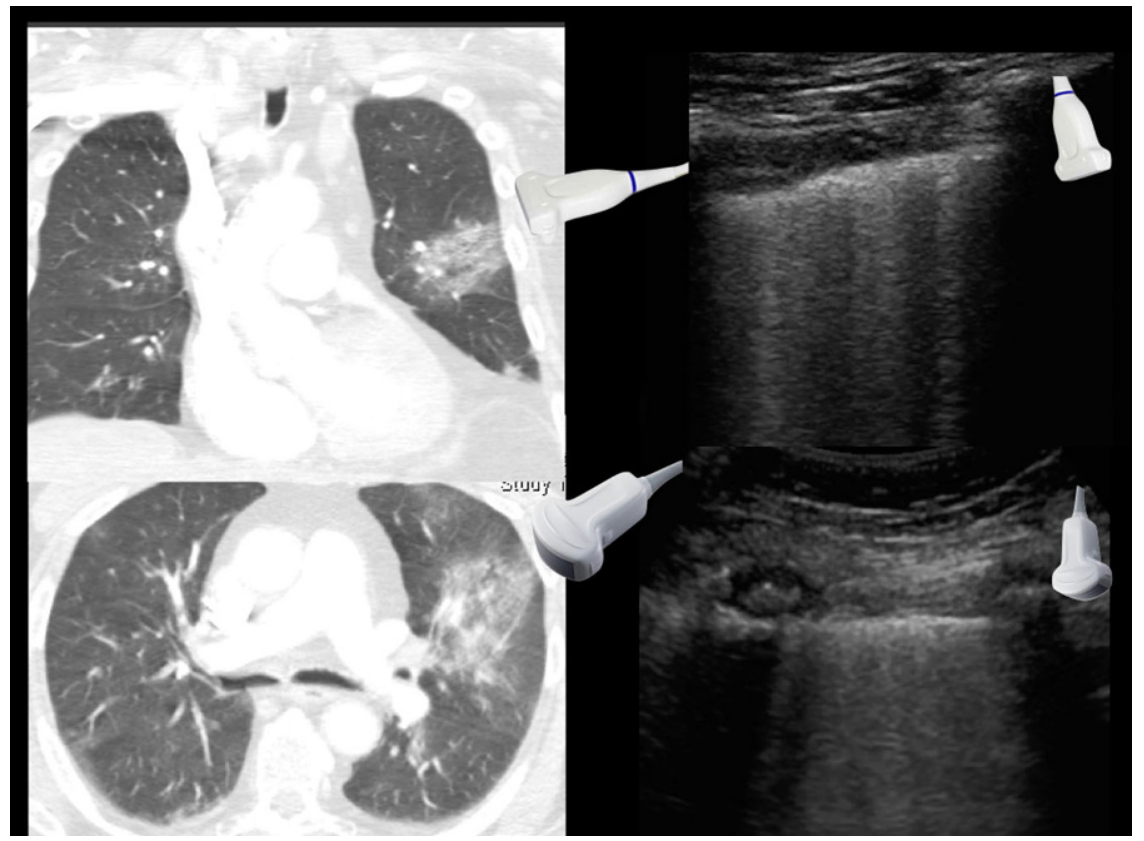

Abb. $7 \Delta$ Muster multipler, von der Pleura ausgehender B-Linien (fokal, regional), die bis an den unteren Bildrand ziehen. Sie bewegen sich mit der Lungenbewegung; Linear- und Konvexschallkopf. (Mit freundl. Genehmigung Dr. M. Schmid, München)

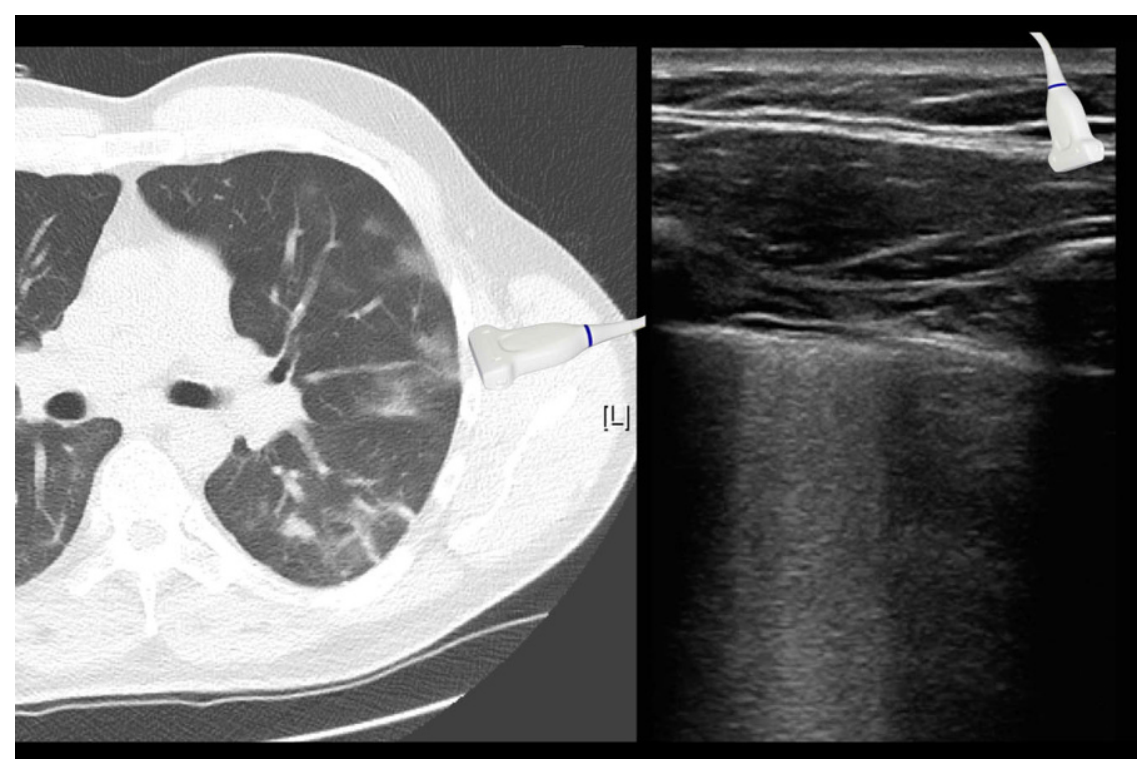

Abb. $8 \Delta$ Muster verschmelzender B-Linien (links) mit angrenzend normalem Lungensonogramm (rechts), sog. „Wasserfallzeichen“ [19]. (Mit freundl. Genehmigung Dr. M. Schmid, München)

Philips, Hamburg) im Vergleich zur normalen Lungenoberfläche vorgestellt (• Abb. 4, 5, 6, 7, 8 und 9; [43]). Diese sind CT-korreliert worden. Hinzu kommen Beispielbefunde mit Qualität der Taschenultraschallgeräteklasse von 7 weiteren Fällen (• Abb. 10).

Filme zu Abbildungen und Untersuchungsweise können über den wissenschaftlichen Literatur vorgestellt wurden, davon 10 von Kindern. Diese wurden detailliert beschrieben, auch für klinische Verlaufsuntersuchungen, und wiesen bei verschiedenen Autorengruppen Gemeinsamkeiten in der Analyse der Muster der Sonogramme auf $[28,48]$. Es gibt zwischenzeitlich eine russische S1- und eine US-Leitlinie [3, 32].

Dennoch sollte man die vorliegende Evidenz für die Lungen- und Thoraxsonographie weiterhin kritisch sehen [16]. Es besteht eine Kontroverse und Diskrepanz bez. der aktuell verfügbaren, kontrollierten Studien und der Erfahrung von Anwendern mit der Methode. Daher sollten vorschnelle Schlussfolgerungen bei Befunden von nun bekannten Mustern vermieden werden [16].

\section{Fazit}

Es gibt in der wissenschaftlichen Literatur zunehmende Evidenz zum Nutzen der Lungen- und Thoraxsonographie bei COVID-19-Pneumonie für Diagnostik und Verlauf.

Autoren aus China, Italien, Spanien, Frankreich, den USA, Brasilien, Russland und Deutschland wiesen auf die Besonderheit des Verfahrens hin und sehen die Lungen- und Thoraxsonographie als Ergänzung zur Diagnostik bei COVID-19Erkrankung [3, 32, 38, 43, 47, 48].

Aufgrund der einfachen Umsetzbarkeit bietet sich neben der Thorax- und Lungensonographie eine „Multiorganuntersuchung" mittels Point-of-CareUltraschall (PoCUS), unter Beachtung der Schutz- und Hygieneempfehlungen während der Pandemie, als Untersuchungsmodalität im Sinne eines „PoCUS“-Protokolls an [3]. Dennoch sollte die Methode aufgrund der begrenzten Datenlagen weiterhin kritisch betrachtet werden, da Sensitivität und Spezifität des Verfahrens noch unklar sind.

Die multimedialen Lernressourcen sollen den Zugang zur Methode und Wissensvermittlung für den Anwender unterstützen.
Als Limitation im speziellen Zusammenhang für COVID-19-Patienten ist unbeschadet der Methode Lungen- und Thoraxsonographie zu beachten, dass bisher zusammen etwas mehr als 200 Fälle in der

Permalink https://www.yumpu.com/s/ tiNYSqf0cBhG8reb aufgerufen werden.

\section{Limitationen}



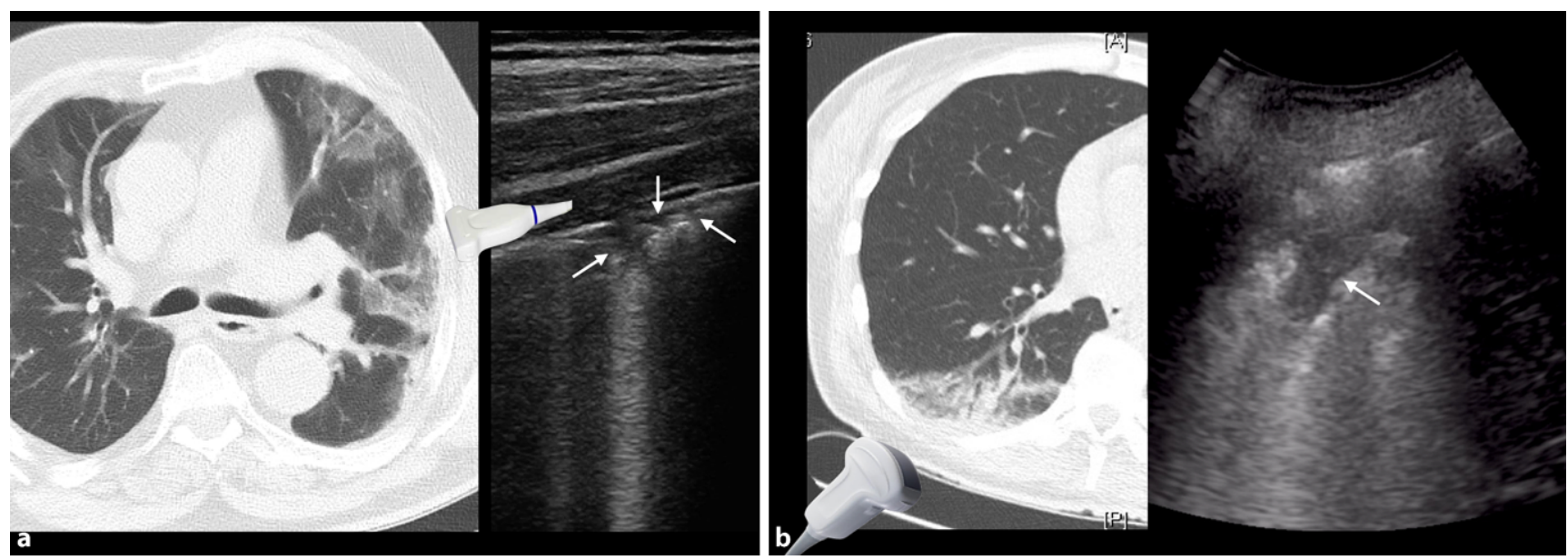

Abb. $9 \Delta$ a Muster kleinerer subpleuraler Konsolidierungen mit Unterbrechung der Pleuralinie; Linearschallkopf. b Muster größerer subpleuraler Konsolidierungen mit weiterhin angrenzenden Aerobronchogrammen, die Pleuralinie ist unterbrochen; Konvexschallkopf. (a, b Mit freundl. Genehmigung Dr. M. Schmid, München)
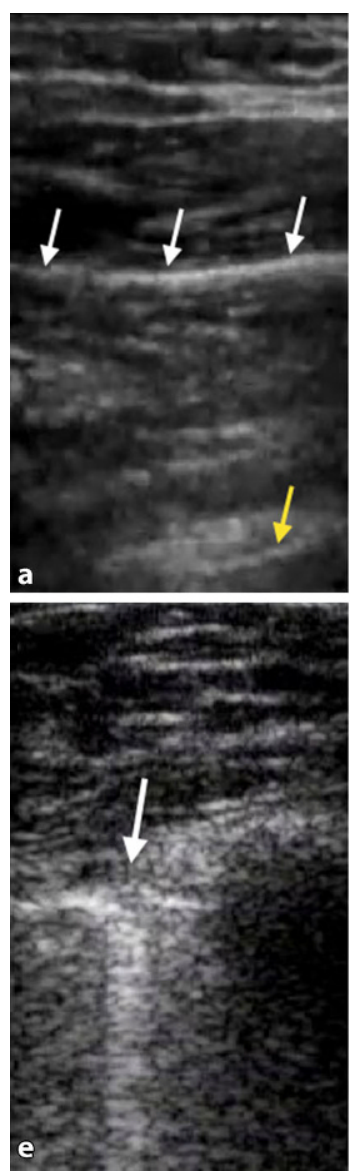
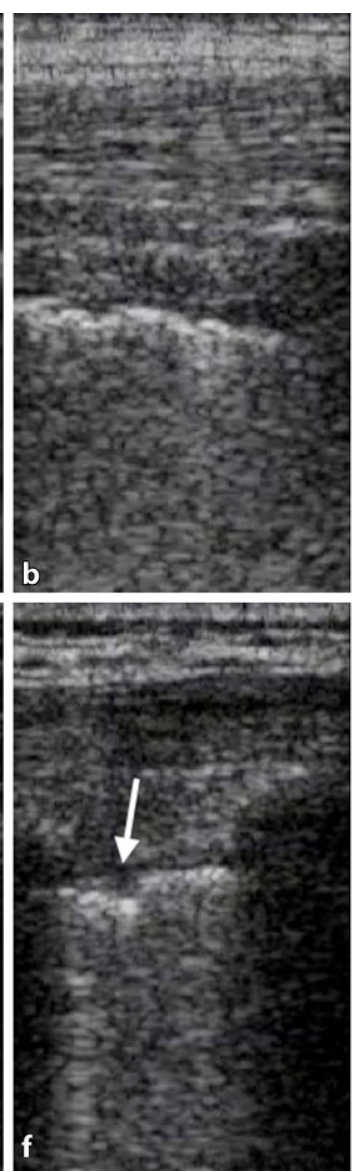
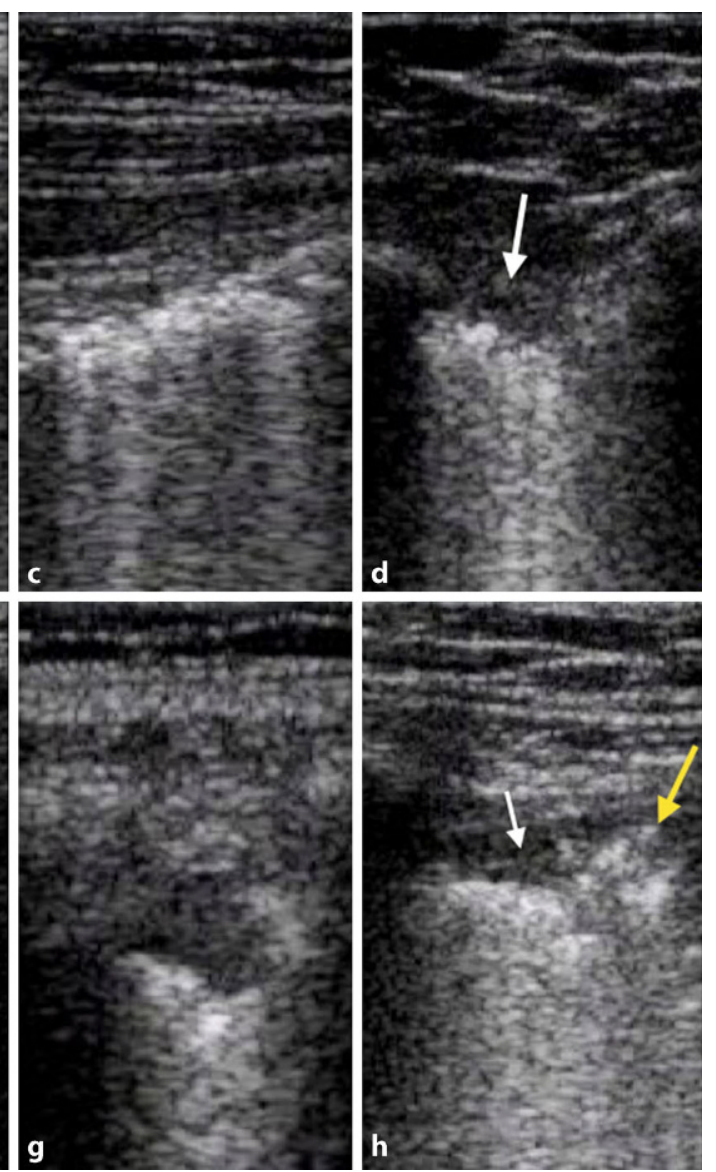

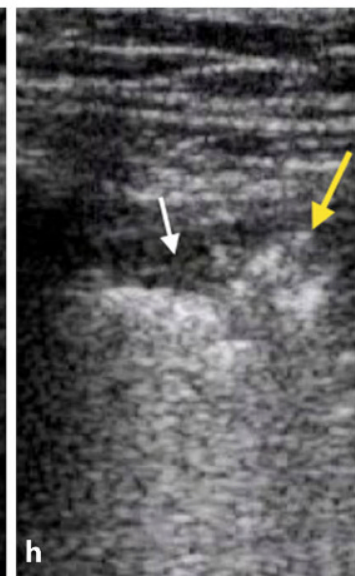

Abb. $10<$ Befunde der Lungenoberfläche mit einem ultramobilen "Hand-held"- oder "Pocketsized"-Ultraschallgerät (Vscan 1.1 GE, Solingen) beim Gesunden (a) und bei COVID-19-Pneumonie $(\mathbf{b}-\mathbf{h})$; a normale Lungenoberfläche (Pleuralinie $=$ Pleura visceralis, weiße Pfeile), Reverberation "A-Linie", gelber Pfeil. b Unruhige Pleuralinie mit Mikrokonsolidierungen und geringen Mengen Flüssigkeit. c Verdickte Pleuralinie mit fokalen, multiplen B-Linien. d Unterbrechung der Pleuralinie (Pfeil) und verschmelzende B-Linien (,Wasserfall“).e,f Kleine Konsolidierungen. g Große Konsolidierung mit Unterbrechung der Pleuralinie. $\mathbf{h}$ Konsolidierung (weißer Pfeil) und Infiltration (gelber Pfeil) mit Aerobronchogramm 


\section{Infobox 3 Lehrmaterial mit Originalbefunden (kostenlos, Auswahl)}

Lernressourcen (deutsch, DE)

- https://CoViD-sono.com/ (DE, offene, unabhängige COVID-19-Fall-Sammlung als Lernressource mit Bildern von DEGUM-Mitgliedern)

- https://www.yumpu.com/s/ tiNYSqf0cBhG8reb (Beispielfilme zur Untersuchungsweise)

- https://www.yumpu.com/de/document/ view/63176982/corona-lungensonoonline-pocket-card-fur-alle https://www. youtube.com/channel/UCd2p84BNC9DKcgK0e3WU4g

- https://dasfoam.org/2020/03/18/derlungen-pocus-in-zeiten-der-corona/

- http://www.yumpu.com/de/SonoABCD (E-Learning Thorax- und Lungensonographie)

Podcasts und Webinare (deutsch und englisch, EN)

- [https://notfall-campus.de/aktuelleinformationen-zu-CoViD-19/] https:// vimeo.com/401334105 (D) https://www. coreultrasound.com/usp_CoViD_2/ (EN)

- https://www.youtube.com/watch? $\mathrm{v}=\mathrm{kWORAYuPK4E \& feature}=$ youtu.be $(\mathrm{EN})$

- https://litfl.com/lung-ultrasound-CoViD19-cases/ (EN)

- http://www.emdocs.net/lungultrasound-in-covid-19/ (Lernen)

Sonogramme - Mustererkennung (englisch)

- https://www.grepmed.com/? $q=C O V I D 19+P O C U S$, http://www. thepocusatlas.com/CoViD19(EN)

- https://twitter.com/giovolpicelli/status/ 1238470459680870401, https:// twitter.com/yaletung (EN) https:// twitter.com/drmarcogarrone/status/ 1240887911949709314(EN)

- https://twitter.com/ThinkingCC/status/ 1241473896676810752 (EN)

- http://www.emdocs.net/wp-content/ uploads/2020/04/Fig-2.png (Schema für Mustererkennung aus [48])

- Bild- und Filmdatenbank, https://CoViDsono.com/ (Hoepfner M, Weskott HP)

\section{Korrespondenzadresse}

Dr. med. Daniel Kiefl

Klinik für Interdisziplinäre Notfallmedizin, Sana Klinikum Offenbach $\mathrm{GmbH}$

Starkenburgring 66, 63069 Offenbach am Main, Deutschland

daniel.kiefl@sana.de

Prof. Dr. Dr. med. habil. Raoul Breitkreutz

Fachbereich/Institut f. Gesundheit \& Soziales (IfGS), FOM Hochschule für Ökonomie \&

Management

Franklinstr. 52, 60486 Frankfurt am Main,

Deutschland

raoul.breitkreutz@fom.de

Danksagung. Die Autoren danken Prof. Th. Helmberger, Institut für Radiologie, Neuroradiologie und minimal-invasive Therapie, München Klinik Bogenhausen für die Nutzung der CT-Originalbefunde und Dr. Daniel Tepperis, Agaplesion Elisabethenstift, Darmstadt für Befunde der $\bullet \mathrm{Abb} .10$

\section{Einhaltung ethischer Richtlinien}

Interessenkonflikt. G. Michels gibt an, persönliche Honorare von den Firmen ZOLL, Sedana Medical, Getinge und Orion Pharma außerhalb der eingereichten Arbeit erhalten zu haben; es besteht kein Interessenkonflikt in Zusammenhang mit der vorliegenden Arbeit. Der Point-of-Care-Ultraschall (PoCUS) im Rahmen von Ausbildungsveranstaltungen für Medizinstudenten oder in einzelnen Seminaren am Institut von D. Damjanovic wurden manchmal mittels der vergünstigten oder kostenlosen Ausleihe von Ultraschallgeräten innerhalb genehmigter Modellprojekte durch folgende Firmen unterstützt: GE Healthcare, Butterfly Network Inc., Philips Deutschland, Fujifilm Sonosite, Clarius, Multimed GmbH. S. Schellhaas ist Gesellschafter der UEC \& Partners I Medical Education $\mathrm{GmbH}$. D.-A. Clevert hat außerhalb der eingereichten Arbeit eingeladene Vorträge für die Firmen Samsung Siemens, Philips, Esaote und Bracco gehalten. R. Breitkreutz erhielt Honorare von Fa. Mindray Medical, Deutschland. D. Kiefl, S. Eisenmann, M. Schmid, C. Ludwig, M. Pin, E. Glöckner, P.-F. Petersen, U. Janssens, M. Fandler, S. Blaschke, M. Geuting, T. Müller, J. Menzel, A. Heinzmann, M. Helm, A. Dinse-Lambracht, M. Bernhard, S. Spethmann und K.F. Stock geben an, dass kein Interessenkonflikt besteht.

Für diesen Beitrag wurden von den Autoren keine Studien an Menschen oder Tieren durchgeführt. Für die aufgeführten Studien gelten die jeweils dort angegebenen ethischen Richtlinien.

\section{Literatur}

1. Ai T et al (2020) Correlation of chest CT and RT-PCR testing in Coronavirus disease 2019 (COVID-19) in China: a report of 1014 cases. Radiology 26:200642

2. Antoch $G$ et al (2020) SARS-CoV-2/COVID-19: Empfehlungen für die Radiologische Versorgung. Rofo 192(5):418-421. https://doi.org/10.1055/a$1149-3625$
3. Johri AM et al (2020) ASE statement on point-ofcare ultrasound during the 2019 novel Coronavirus pandemic. J Am Soc Echocardiogr. https://doi.org/ 10.1016/j.echo.2020.04.017

4. Bedetti Getal (2006) Evaluation of ultrasound lung comets by hand-held echocardiogr. Cardiovasc Ultrasound 4:34

5. Buonsenso D et al (2020) COVID-19 outbreak: less stethoscope, more ultrasound. Lancet Respir Med 8(5):e27

6. Buonsenso D et al (2020) Point-of-care lung ultrasound findings in novel coronavirus disease19 pnemoniae: a case report and potential applications during COVID-19 outbreak. Eur Rev Med Pharmacol Sci 24:2776-2780

7. Breitkreutz R, Dutiné $M$ et al (2013) Thorax, trachea, and lung ultrasonography in emergency and critical care medicine: assessment of an objective structured training concept. Emerg Med Int 2013:312758. https://doi.org/10.1155/2013/ 312758

8. Clevert DA, Schroeder I, Sabel B (2020) Atemnot und Ultraschall. Bayer Arztebl 6:204

9. Copetti R et al (2008) Chest sonography: a useful tool to differentiate acute cardiogenic pulmonary edema from acute respiratory distress syndrome. Cardiovasc Ultrasound 6:16

10. Cox EGM et al (2020) Should the ultrasound probe replace your stethoscope? A SICS-I substudy comparing lung ultrasound and pulmonary auscultation in the critically ill. Crit Care 24:14. https://doi.org/10.1186/s13054-019-2719-8

11. Dargent A et al (2020) Lung ultrasound score to monitor COVID-19 pneumonia progression in patients with ARDS. PLoS ONE 15(7):e236312. https://doi.org/10.1371/journal.pone.0236312

12. Davenport $L$ (2020) Under pressure, one Italian doctor triages by ultrasound. https://www. medscape.com/viewarticle/927470. Zugegriffen: 24. März 2020

13. EPA (2020) Desinfektionsmittel. https://www.epa. gov/pesticide-registration/list- $n$-disinfectantsuse-against-sars-cov-2. Zugegriffen: 24. Juli 2020

14. Duclos G, Lopez A, Leone M, Zieleskiewicz L (2020) "No dose" lung ultrasound correlation with "low dose" CT scan for early diagnosis of SARS-CoV-2 pneumonia. Intensive Care Med 4:1-2. https://doi org/10.1007/s00134-020-06058-7

15. Gargani L, Volpicelli G (2014) How I do it: lung ultrasound. Cardiovasc Ultrasound 12:25

16. Gargani L et al (2020) Why, when, and how to use lung ultrasound during the COVID-19 pandemic: enthusiasm and caution. Eur Heart J Cardiovasc Imaging. https://doi.org/10.1093/ehjci/jeaa163

17. Guan WJ et al (2020) Clinical characteristics of Coronavirus disease 2019 in China. N Engl J Med 382:1708-1720. https://doi.org/10.1056/ NEJMoa2002032

18. Hach-Wunderle V et al (2016) S2k Leitlinie. Vasa 45(90):1-48

19. Huang $Y$ et al (2020) A preliminary study on the ultrasonic manifestations of peripulmonary lesions of non-critical novel coronavirus pneumonia (COVID-19). https://papers.ssrn.com/sol3/papers. cfm?abstract_id=3544750 https://doi.org/10. 21203/rs.2.24369/v1.Zugegriffen:1.Aug. 2020

20. RKI (2020) Hygiene bei COVID-19. https:// www.rki.de/DE/Content/InfAZ/N/Neuartiges Coronavirus/Hygiene.html. Zugegriffen: 24. Juli 2020

21. No Authors listed (2011) Hygiene beiUltraschallanwendungen. Bundesgesundheitsbl 54:1135-1144

22. Inciardi RM et al (2020) Cardiac involvement in a patient with Coronavirus disease 2019. JAMA 
Cardiol 5(7):819-824. https://doi.org/10.1001/ jamacardio.2020.1096

23. JiL, CaoC, Lv Q, LiY, Xie M(2020) Serial bedsidelung ultrasonography in a critically ill COVID-19 patient. QJM. https://doi.org/10.1093/qjmed/hcaa141

24. Kluge S et al (2020) Empfehlungen zur intensivmedizinischen Therapie von Patienten mit COVID-19 (S1-Leitlinie (V3 vom 21.7.2020. AWMF-RegisterNr. 113/001))

25. Lichtenstein D, Goldstein I, Mourgeon E, Cluzel P, Grenier P, Rouby J-J (2004) Comparative diagnostic performances of auscultation, chest radiography, and lung ultrasonography in acute respiratory distress syndrome. Anesthesiology 100:9-15

26. Llitjos JF et al (2020) High incidence of venous thromboembolic events in anticoagulated severe COVID-19 patients. JThromb Haemost. https://doi. org/10.1111/jth.14869

27. Liu Y, Ning Z, Chen Y et al (2020) Aerodynamic analysis of SARS-CoV-2 in two Wuhan hospitals. Nature. https://doi.org/10.1038/s41586-020 2271-3

28. Lomoro P et al (2020) COVID-19 pneumonia manifestations at the admission on chest ultrasound, radiographs, and $\mathrm{CT}$ : single-center study and comprehensive radiologic literature review. Eur J RadiolOpen 7:100231

29. Lu W et al (2020) A clinical study of noninvasive assessment of lung lesions in patients with Coronavirus disease-19 (COVID-19) by bedside ultrasound. Ultraschall Med. https://doi.org/10. 1055/a-1154-8795

30. Mathis $G$ et al (2005) Thoracic ultrasound for diagnosing pulmonary embolism: a prospective multicenter study of 352 patients. Chest 128:1531-1538

31. Mathis $G$ et al (1990) Lung infarcts detected with ultrasonography. Ultraschall Med 11(6):281-283

32. Митьков ВВ, Сафонов ДВ et al (2020) Consensus guidelines of Russian society of radiology (RSR) and Russian association of specialists in ultrasound diagnostics in medicine (RASUDM) "role of imaging (X-ray, CT and US) in diagnosis of COVID-19 pneumonia" (version 2). Vestn Rentgenol Radiol 101(2):72-89. https://doi. org/10.24835/1607-0771-2020-1-24-45

33. Mongodi S, Orlando A, Arisi E et al (2020) Lung ultrasound in patients with acute respiratory failure reduces conventional imaging and health care provider exposure to COVID-19. Ultrasound Med Biol. https://doi.org/10.1016/j.ultrasmedbio. 2020.04.033

34. Müller T et al (2018) DEGUM-Empfehlungen zur Hygiene in Sonografie und Endosonografie. Ultraschall Med 39(03):284-303

35. Musolino AM, Supino MC, Buonsenso D et al (2020) Lung ultrasound in children with COVID-19: preliminary findings. Lung ultrasound study team for pediatric COVID-19 (ROMULUS COVID team). Ultrasound Med Biol. https://doi.org/10.1016/j. ultrasmedbio.2020.04.026

36. Neskovic AN et al (2018) Focus cardiac ultrasound core curriculum and core syllabus of the European association of cardiovascular imaging. Eur Heart J Cardiovasc Imaging 19:475-481. https://doi.org/ 10.1093/ehjci/jey006

37. Peng QY et al (2020) Findings of lung ultrasonography of novel corona virus pneumonia during the 2019-2020 epidemic. Intensive Care Med 46(5):849-850. https://doi.org/10.1007/s00134020-05996-6

38. Pereira EF Jr et al (2020) http://winfocus.org/wpcontent/uploads/2020/03/WINFOCUS_CoViD19_ Brazil-English-1.pdf.Zugegriffen: 24. Juli 2020
39. Piscaglia F et al (2020) Benefits, open questions and challenges of the use of ultrasound in the COVID-19 pandemic era. The views of a panel of worldwide international experts. Ultraschall Med. https://doi.org/10.1055/a-1149-9872

40. Poggiali E et al (2020) Can lung US help critical care clinicians in the early diagnosis of novel Coronavirus (COVID-19) pneumonia? Radiology 295(3):E6. https://doi.org/10.1148/ radiol.2020200847

41. Reissig A et al (2001) Sonography of lung and pleura in pulmonary embolism: sonomorphologic characterization and comparison with spiral CT scanning. Chest 120(6):1977-1983

42. AWMF online (2018) S3 Leitlinie zu Pneumothorax. https://pneumologie.de/fileadmin/user upload/010-007I_S3_Spontanpneumothoraxpostinterventioneller-Pneumothorax-DiagnostikTherapie_2018-03.pdf.Zugegriffen:24.Juli 2020

43. Schmid M Aktuelle Informationen zu COVID-19. https://notfall-campus.de/aktuelleinformationen-zu-CoViD-19/. Zugegriffen: 24. Juli 2020 (Webinar)

44. Schmid M (2020) CoViD-19 in der Notaufnahme. Bayer Arztebl 6:205

45. Schmid M (2020) Lung ultrasound findings in COVID-19 pneumonia. Dtsch Arztebl Int 117:335. https://doi.org/10.3238/arztebl.2020.0335

46. Shi $\mathrm{H}$ et al (2020) Radiological findings from 81 patients with COVID-19 pneumonia in Wuhan: a descriptive study. Lancet Infect Dis. https://doi. org/10.1016/S1473-3099(20)30086-4

47. Shokoohi H, Duggan NM, García-de-Casasola Sánchez Getal (2020) Lung ultrasound monitoring in patientswith COVID-19 on home isolation. Am J Emerg Med. https://doi.org/10.1016/j.ajem.2020. 05.079

48. Smith MJ et al (2020) Point-of-care lung ultrasound in patients with COVID-19-a narrative review. Anaesthesia.https://doi.org/10.1111/anae.15082

49. Soldati $G$ et al (2020) Proposal for international standardization of the use of lung ultrasound for COVID-19 patients; a simple, quantitative, reproducible method. J Ultrasound Med. https:// doi.org/10.1002/jum.15285

50. Soldati $G$ et al (2020) Is there a role for lung ultrasound during the COVID-19 pandemic? J Ultrasound Med 39(7):1459-1462. https://doi. org/10.1002/jum.15284

51. Soldati G et al (2020) Contrast-enhanced ultrasound in patients with COVID-19: pneumonia, acute respiratory distress syndrome, or something else? J Ultrasound Med. https://doi.org/10.1002/ jum.15338

52. Soummer A et al (2012) Ultrasound assessment of lung aeration loss during a successful weaning trial predicts postextubation distress. Crit Care Med 40(7):2064-2072

53. Stock KF, Mathis G, Horn R (2020) Lungenultraschallprotokoll vom 6.4.2020 der DEGUM / ÖGUM / SGUM. https://www.degum.de/ fileadmin/dokumente/service/Downloads/Poster_A4-Lungenultraschall-Proto-koll_DEGUM SGUM_OEGM_V3_06042020_Print_digital_NEU. pdf. Zugegriffen: 24. Juli 2020

54. Stock KF, Treiber M, Braren R (2020) Diagnose mit dem „Ultraschall-Stethoskop“. Bayer Arztebl 6:206

55. Tan $G$ et al (2020) Use of lung ultrasound to differenciate coronavirus disease 2019 (COVID-19) pneumonia from community-acquired pneumonia. Ultrasound Med Biol. https://doi.org/10.1016/ j.ultrasmedbio.2020.05.006

56. Tasci O, Hatipoglu ON, Cagli B, Ermis V (2016) Sonography of the chest using linear-array versus sector transducers: correlation with auscultation chest radiography, and computed tomography. JClin Ultrasound 44:383-389

57. Tavazzi G, Civardi L, Caneva L, Mongodi S, Mojoli F (2020) Thrombotic events in SARS-Cov 2 patients: an urgent call for ultrasound screening. Intensive Care Med. https://doi.org/10.1007/s00134-02006040-3

58. Tee $A$ et al (2020) Contrast-enhanced ultrasound (CEUS) of the lung reveals multiple areas of microthrombi in a COVID-19 patient. Intensive Care Med. https://doi.org/10.1007/s00134-020 06085-4

59. Torino C, Gargani L, Sicari R, Letachowicz K, Ekart R Fliser D et al (2016) The agreement between auscultation and lung ultrasound in hemodialysis patients: the LUST study. Clin J Am Soc Nephrol 11:2005-2011

60. Tung-Chen $Y$ (2020as) Lung ultrasound in the monitoring of COVID-19 infection. Clin Med. https://doi.org/10.7861/clinmed.2020-0123

61. van Doremalen $\mathrm{N}$ et al (2020) Aerosol and surface stability of SARS-coV-2 as compared with SARScoV-1. N Engl J Med. https://doi.org/10.1056/ NEJMc2004973

62. Vetrugno Let al (2020) Our Italian experience using lung ultrasound for identification, grading and serial follow-up of severity of lung involvement for management of patients with COVID-19. Echocardiography 37(4):625-627. https://doi.org/ 10.1111/echo.14664

63. Via G et al (2014) International evidence-based recommendations for focused cardiac ultrasound. J Am Soc Echocardiogr 27(7):683.e1-683.e33. https://doi.org/10.1016/j.echo.2014.05.001

64. Volpicelli G, Gargani L (2020) Sonographic signs and patterns of COVID-19 pneumonia. Ultrasound J 12(1):22. https://doi.org/10.1186/ s13089-020-00171-w

65. Volpicelli G et al (2012) Internat. evidencebased recommendations for point-of-care lung ultrasound. Intensive Care Med 38(4):577-591

66. Volpicelli $G$ et al (2020) What's new in lung ultrasound during the COVID-19 pandemic. Intensive Care Med. https://doi.org/10.1007/ s00134-020-06048-9

67. Wichmann D, Sperhake JP, Lütgehetmann $M$, Steurer S, Edler C, Heinemann A, Heinrich F et al (2020) Autopsy findings and venous thromboembolism in patients with COVID-19. Ann Intern Med. https://doi.org/10.7326/M20-2003

68. Xing $C$ et al (2020) Lung ultrasound findings in patients with COVID-19 pneumonia. Crit Care 24:174. https://doi.org/10.1186/s13054-02002876-9

69. Zotzmann Vetal (2020) Are subpleural consolidations indicators for segmental pulmonary embolism in COVID-19? Intensive Care Med. https://doi.org/ 10.1007/s00134-020-06044-z 


\section{Affiliations}

' Klinik für Interdisziplinäre Notfallmedizin, Sana Klinikum Offenbach GmbH, Offenbach am Main, Deutschland; ${ }^{2}$ Schwerpunkt Pneumologie, Universitätsklinikum Halle (Saale), Halle (Saale), Deutschland; ${ }^{3}$ Klinik für Akut- und Notfallmedizin, St.-Antonius-Hospital gGmbH, Eschweiler, Deutschland; ${ }^{4}$ Klinik für Gastroenterologie, Pneumologie, Internistische Akut- und Intensivmedizin, München Klinik Harlaching, München, Deutschland; ${ }^{5}$ Klinik für Thoraxchirurgie, Florence-Nightingale-Krankenhaus der Kaiserswerther Diakonie, Düsseldorf, Deutschland; ${ }^{6}$ Zentrale interdisziplinäre Notaufnahme und Aufnahmebereich, Florence-Nightingale-Krankenhaus der Kaiserswerther Diakonie, Düsseldorf, Deutschland; ' ${ }^{7}$ Klinik für Gastroenterologie, Endokrinologie und Zentrale Notaufnahme Nord, Klinikum Nürnberg Nord, Paracelsus Medizinische Privatuniversität, Nürnberg, Deutschland; ${ }^{8}$ Zentrale Notaufnahme, Klinikum Frankfurt Höchst, Höchst, Deutschland; ${ }^{9}$ Klinik für Herz- und Gefäßchirurgie, Universitäts-Herzzentrum Freiburg - Bad Krozingen, Freiburg, Deutschland; ${ }^{10}$ Hausarztpraxis Dr. Schellhaas, Groß-Bieberau, Deutschland; " Klinik für Innere Medizin und Internistische Intensivmedizin, St.-Antonius-Hospital gGmbH, Eschweiler, Deutschland; ${ }^{12}$ Interdisziplinäre Notaufnahme, Klinikum Bamberg, Bamberg, Deutschland; ${ }^{13}$ Interdisziplinäre Notaufnahme, Universitätsmedizin Göttingen, Göttingen, Deutschland; ${ }^{14}$ Abteilung Anästhesie, Vulpius Klinik, Bad Rappenau, Deutschland; ${ }^{15}$ Medizinische Klinik II, St. Josefs-Hospital Wiesbaden GmbH, Wiesbaden, Deutschland; ${ }^{16}$ DEGUM Kursleiter Innere Medizin, Endosonographie, Medizinische Klinik II, Klinikum Ingolstadt, Ingolstadt, Deutschland; ${ }^{17}$ Medizinische Klinik, Klinikum Am Steinenberg, Reutlingen, Deutschland; ${ }^{18}$ Abteilung X (Anästhesie), Bundeswehrkrankenhaus, Ulm, Deutschland; ${ }^{19}$ Interdisziplinäres Notfallzentrum, Trägergesellschaft Kliniken Aurich-Emden-Norden, Aurich-Emden-Norden, Deutschland; ${ }^{20}$ Zentrale Notaufnahme, Universitätsklinikum Düsseldorf, Düsseldorf, Deutschland; ${ }^{21}$ Medizinische Klinik A Kardiologie, Angiologie, Nephrologie, internistische Intensivmedizin, Medizinische Hochschule Brandenburg, Campus Ruppiner Kliniken, Neuruppin, Deutschland; ${ }^{22}$ Nephrologisches Ultraschalllabor, TU München, München, Deutschland; ${ }^{23}$ Klinik und Poliklinik für Radiologie, Interdisziplinäres Ultraschallzentrum, Universität München, Klinikum Großhadern, München, Deutschland; ${ }^{24}$ Fachbereich/Institut f. Gesundheit \& Soziales (IfGS), FOM Hochschule für Ökonomie \& Management, Frankfurt am Main, Deutschland 